\title{
EVALUACIÓN DEL CONTENIDO DE ANTOCIANINAS DE TRES ACCESIONES DE OCA (OXALIS TUBEROSA) EN CONDICIONES FRESCAS Y COCIDAS DEL DEPARTAMENTO DE JUNÍN
}

\section{ASSESSMENT ANTHOCYANIN CONTENT OF THREE ACCESSIONS OF OCA (OXALIS TUBEROSA) IN COOL AND COOKED THE DEPARTMENT OF JUNIN}

\author{
N. Gamarra Mendoza ${ }^{1}$ \\ C. Girón2;B. Roque Lima3, J. Díaz Romero3 \\ Facultad Ingeniería en Industrias Alimentarias
}

\section{RESUMEN}

Se realizó el cultivo de las accesiones de oca en el anexo de Masacancha, provincia de Jauja, en coordinación con la oficina de Recursos Genéticos del INIA- EESA-Huancayo, el cultivo tuvo un ciclo vegetativo de 290 días al cabo de este tiempo se realizó la cosecha y seguidamente los análisis físicos y fisicoquímicos de las accesiones púrpura, rojo, amarillo-rosado. Las muestras fueron analizadas en estado fresco y cocido, en fresco se evaluó el color, el tamaño, peso y forma. Asimismo, se determinó el índice de madurez fisiológica en base al contenido de sólidos solubles y acidez total, también se determinó el valor de pH como un indicador del grado de acidez y de madurez de los tubérculos. La extracción de antocianinas se realizó utilizando etanol al 80\% acidificado, purificado con C18 SPE, evaporado y resuspendido en agua acidulada $(0,1 \%)$, seguidamente fue analizado el contenido de polifenoles totales, antocianinas y la capacidad antioxidante de estos compuestos. En estado fresco, la oca púrpura muestra un mayor contenido de 1.2 y 3.5 veces que las accesiones rojo peruanito y rosado. Respecto al contenido de antocianinas monoméricas (mg/100 g muestra), la oca purpura fresca muestra un mayor nivel de 1.2 y 2.1 veces mayor que las otras accesiones. Después de la cocción por ebullición, las tres accesiones muestran un reducción significativa $(P<0.05)$ de polifenoles y capacidad antioxidante, estos compuestos son muy termolábiles a temperaturas por encima de $40^{\circ} \mathrm{C}$. Los cromatogramas de la oca púrpura muestra dos picos con área y altura significativa y con tiempos de retención de 8,4 y 4,5 min.

Palabras clave: Antocianinas, antocianidinas, capacidad antioxidante, polifenoles, oca

\begin{abstract}
Oca accessions cultivation was performed in the annex Masacancha, Jauja Province, in coordination with the Office of Genetic Resources INIA-EESA-Huancayo, cultivation had a growing season of 290 days after this time was harvest and then the physical and physicochemical analysis of accessions purple, red, yellow, pink. Samples were analyzed in fresh and cooked fresh evaluated the color, the size, weight and shape. It was also found physiological maturity index based on the content of soluble solids and total acidity was also determined $\mathrm{pH}$ value as an indicator of the degree of acidity and maturity of the tubers. Extraction of anthocyanins was performed using $80 \%$ ethanol acidified with C18 SPE purified, evaporated and resuspended in acidified water $(0.1 \%)$, then analyzed the content of total polyphenols, anthocyanins and antioxidant capacity of these compounds. While fresh, purple goose shows a higher content of 1.2 and 3.5 times that accessions Peruanito red and pink. Regarding the monomeric anthocyanin content (mg/100 g sample), the fresh purple goose shows a higher level of 1.2 and 2.1 times larger than the other accessions. After cooking by boiling, the three accessions showed a significant reduction $(P<0.05)$ antioxidant polyphenols and these compounds are very thermolabile at temperatures above $40^{\circ} \mathrm{C}$. The chromatograms of the purple goose shows two peaks with significant area and height and with retention times 8.4 and $4.5 \mathrm{~min}$.
\end{abstract}

Key words: Antocianinas, antocianidinas, antirust capacity, polifenoles, oca

1 nngamarram@hotmail.com 


\section{INTRODUCCIÓN}

Los polifenoles son metabolitos secundarios presentes en plantas y actúan como protectores contra el estrés biótico y abiótico. Las antocianinas son la mayor clase de polifenoles que proporcionan el color rojo, púrpura y azúl de muchas frutas, hortalizas y tubérculos (Hager et al., 2008). Actualmente las antocianinas son de interés en la industria de alimentos como colorantes alimenticios seguros, saludables y efectivos, y están disponibles en fuentes vegetales. Las antocianinas tienen un potencial antioxidante significativa, bloquean la reacción de radicales oxigenados libres (ORAC) (Wang et al. 1997; Tsuda et al. 2000), e inhiben la oxidación del LDL (Kahkonen \& Heinonen, 2003), estos descubrimientos sugieren que el contenido de antocianinas en bayas, uvas y papa morada puede proporcionar posibles beneficios a la salud, tales como la reducción de CHD (Zern et al. 2005), actividad anti-inflamatoria (Afaq et al. 2005) y anticancerígena (Hagiwara et al. 2002). Estudios epidemiológicos han indicado que la ingesta de frutas y vegetales ricos en antocianinas están relacionados con los beneficios de la salud. Muchos de los cultivos andinos, dependiendo de la variedad genética, pueden contener considerables cantidades de antocianinas. Dentro de la especie de oca, existen accesiones de colores púrpura, rojo, rosado y de colores matizados, que revelan un contenido potencial de antocianinas en condiciones frescas, pero estos tubérculos por lo general son consumidos cocidos en ebullición y se desconoce el efecto de la temperatura de cocción sobre los polifenoles y su capacidad antioxidante. Este estudio evaluó los cambios de estos compuestos bioactivos de oca sometidos a cocción y en condiciones fresca.

\section{MATERIALES Y MÉTODOS}

\section{Lugar de ejecución}

Se realizó en el laboratorio de Química de Alimentos de la Facultad de Ing.de Industrias Alimentarias.

\section{Materiales}

Material biológico

Género:

\section{Especie: \\ Oxalis tuberosa \\ Nombre común: \\ Oca}

Se utilizó tres accesiones de oca (morado, rojo y rosado), procedente de las parcelas experimentales de cultivo del INIA - Hyo, en el anexo de Masacancha de la provincia de Jauja.

\section{Materiales equipos e instrumentos analíticos de laboratorio}

Agitador magnético, Balanza Analítica, Estufa esterilizadora, Agitador orbital Shaker Potenciómetro rango de pH (0.00-14.00); Espectrofotómetro UV - VIS AUTO - LaboMed INC, Centrifuga refrigerada MPW $-350 \mathrm{R}$, Cromatógrafo líquido - HPLC: Incubadora marca Memmert, Rotavapor R - 200/205 BÜCHI, Congeladora ULTRA FRÍO UF 19, Vortex MAXI MIX II - Thermo Scientific, Micropipetas de: 10- $200 \mu \mathrm{l} ; 100-1000$ $\mu \mathrm{l}$; 1000-5000 $\mu \mathrm{l}$., Campana desecadora, Equipo para titulación.

\section{Insumos y reactivos}

Patrón de antocianidinas (cianidina, pelargonina, petunidina), etanol, acetonitrilo, ácido acético, ácido fosfórico, metanol y agua de grado cromatográfico (HPLC), etanol absoluto p.a., tetra cloruro de carbono p.a., hexano p.a., hidróxido de potasio metanólico (40\%), sulfato de sodio (10\%), carbón activado, ácido clorhídrico Q.P., ácido sulfúrico Q.P. anaranjado de metilo p.a. ácido bórico p.a. Verde de bromocresol p.a. Hexano p.a. dicromato de potasio, rojo de metilo p.a.

\section{Determinación del índice de madurez}

Esta determinación se basa en el cociente de la relación entre el porcentaje de los sólidos solubles (\% S.S) y la acidez titulable. Estas características fueron determinados anteriormente por métodos físicos y químicos, respectivamente. El índice de madurez es inversamente proporcional a la cantidad o concentración de ácidos en la muestra, lo cual expresa la madurez fisiológica de la muestra en estudio. 


$$
\text { Índice de Madurez }(\mathrm{IM})=\frac{\% \text { S.S. }}{\% \text { Acidez titulable }}
$$

\section{Extracción y cuantificación de antocianinas}

Se realizó la extracción de polifenoles totales con etanol acidificado al $80 \%$ y se cuantificó por espectrofotometría el contenido de polifenoles, antocianinas monoméricas (Giustin y Wrolstad, 2001) y capacidad antioxidante (Brand-Williams et al., 1995) con modificaciones, se realizó tanto en muestra fresca y cocida, esta última fue sometido a ebullición por 30 min. a temperatura constante, empacado en papel aluminio a fin de evitar el contacto directo con el agua de cocción. Los extractos previamente purificados con una columna C18 SPE, se sometieron a un análisis de cromatografía líquida de alta eficiencia (Handbook of Food Analytical Chemistry, 2008; De Sousa, 2010).

\section{RESULTADOS Y DISCUSIÓN}

\section{Determinación del índice de madurez}

En el Cuadro 1, se muestra los resultados de la determinación del índice de madurez (IM) de las tres accesiones de oca (figura 1). La oca roja presenta un mayor valor de IM de aproximadamente de 1,6 veces comparado a las muestras púrpura y amarillo-rosado, se deduce que la síntesis de sólidos solubles fue mayor en relación al decrecimiento del porcentaje de acidez total, por lo general la madurez fisiológica de frutas, hortalizas y tubérculos va asociado al incremento de sólidos solubles entre ellos predominan los azúcares sencillos, paralelamente en frutos coloreados se intensifica el color, que también es un indicador de maduración de los vegetales para realizar la cosecha. En general las accesiones de oca, después de la cosecha y cuando están en almacenamiento 0 expuestos al, sol se incrementa el contenido de sólidos solubles (azucares) en consecuencia el índice de madurez se ve incrementado, ya que este valor está en relación directa a la cantidad de sólidos solubles. Estudios realizados en 21 accesiones de oca boliviana, durante 21 días de soleado determinaron (Irigoyen y Guidi, 2002)11 el contenido de sólidos solubles de 6 a $8^{\circ}$ Brix.
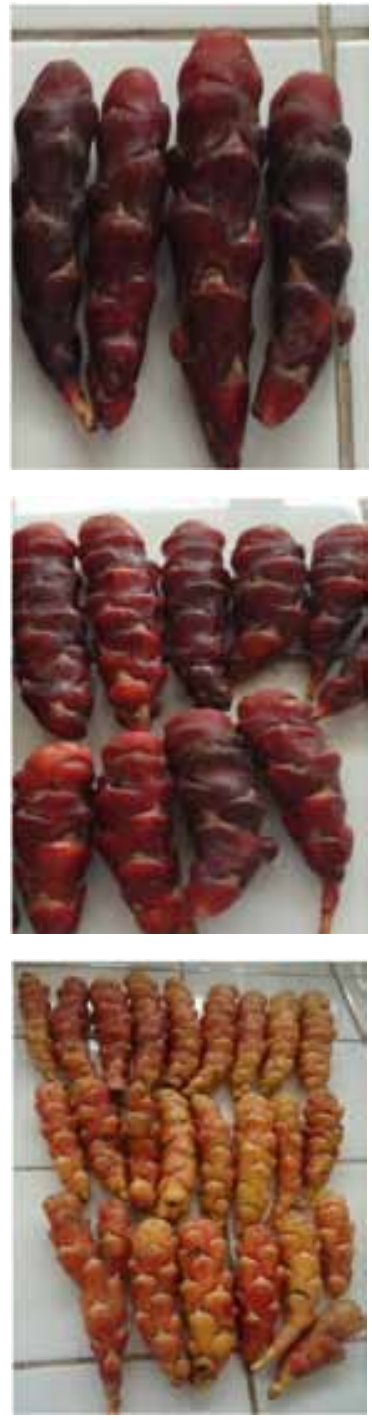

a) Oca púrpura

b) Oca roja peruanito

c) Oca rosada 
Figura 1. Identificación del color de las accesiones de ocas

Cuadro 1. Determinación del índice de madurez de tres accesiones de Oca

\begin{tabular}{lc}
\hline $\begin{array}{c}\text { Accesiones de oca } \\
\text { fresca }\end{array}$ & $\begin{array}{c}\text { Índice de madurez } \\
\text { (\%S.S/\% acidez total) }\end{array}$ \\
\hline Púrpura & 49.12 \\
Roja & 86.36 \\
Amarillo - rosado & 53.03 \\
\hline
\end{tabular}

\section{Extracción y cuantificación de antocianinas}

\section{Determinación de polifenoles totales, antocianinas monoméricas y capacidad antioxidante de tres variedades de oca fresca y cocida}

En el cuadro 2, se muestra el contenido de polifenoles totales y su actividad antioxidante de las tres accesiones de oca en condiciones frescas. En el cual se observa que la oca púrpura muestra un mayor contenido de polifenoles totales de 1,2 y 3,5 veces respecto a la oca roja peruanita y oca rosada, respectivamente. A simple vista esta accesión muestra una distribución de pigmentos superficial e internamente en la corteza y médula. Chirinos, et al (2008) determinaron el contenido de fenoles totales en mashua de la cuarta extracción y obtuvieron $40.5 \pm 9.4 \mathrm{mg} \mathrm{GAE} / 100 \mathrm{~g}$ muestra, siendo menor al de las tres accesiones de oca. En condiciones cocidas la cantidad de polifenoles (mg GAE/100 g muestra) se reduce en 47,$6 ; 39,6$ y $14,3 \%$ de oca púrpura, roja y rosada, respectivamente, pero la oca púrpura mantiene en más del $50 \%$ y en cantidad en relación a las otras accesiones. El efecto de la cocción a temperatura de ebullición es notorio a simple vista y se confirma con la cuantificación química, ya que las accesiones de oca mostraron una disminución de la pigmentación, ya que el agua de cocción quedó coloreado en mayor intensidad en el de oca púrpura, los polifenoles son solubles en solventes polares, asimismo son termolábiles a temperaturas mayores de $40^{\circ} \mathrm{C}$. Natella et al. (2008) evaluaron el efecto de cocción tradicional y por microondas del contenido de compuestos fenólicos y capacidad antioxidante de siete vegetales, donde el coliflor, espinaca, guisantes y acelgas mostraron un significativo decrecimiento en el contenido de polifenoles totales. Respecto al contenido de antocianinas monoméricas $(\mathrm{mg} / 100 \mathrm{~g}$ muestra), la oca púrpura en estado fresco muestra un mayor nivel, siendo de 1.2 y 2.1 veces mayor que las accesiones roja peruanita y rosada, en estado cocido se reduce pero mantiene una cantidad considerable de 1,8 y 6,3 veces mayor que oca roja y rosada. La composición de las antocianinas en uvas y su bagazo difieren de acuerdo a la especie, cultivar, madurez y condiciones climáticas (Mattivi et al., 2006; Mazza, 1995; Muñoz-Espada et al., 2004; Rizzon et al., 1998).

Cuadro 2. Polifenoles totales, antocianinas monoméricas y capacidad antioxidante de oca fresca y cocida

\begin{tabular}{|c|c|c|c|c|c|c|}
\hline \multirow[t]{2}{*}{ Características } & \multicolumn{2}{|c|}{ Oca Púrpura } & \multicolumn{2}{|c|}{ Oca Roja Peruanita } & \multicolumn{2}{|c|}{ Oca Rosada } \\
\hline & Fresco & Cocido & Fresco & Cocido & Fresco & $\begin{array}{c}\text { Coci } \\
\text { do }\end{array}$ \\
\hline $\begin{array}{l}\text { Polifenoles totales } \\
\text { (mg GAE/100 g } \\
\text { muestra oca) }\end{array}$ & 3588.4 & 1707.8 & 2949.1 & 1166.75 & 1034.3 & $\begin{array}{c}148 . \\
0\end{array}$ \\
\hline $\begin{array}{l}\text { Antocianinas } \\
\text { monoméricas } \\
\text { (mg/100 g muestra) }\end{array}$ & 15.12 & 5.7 & 11.74 & 3.25 & 7.2 & 0.9 \\
\hline $\begin{array}{l}\text { Capacidad } \\
\text { antioxidante ( } \mu \mathrm{mol} \\
\text { TE/100 g muestra) }\end{array}$ & 1732,0 & 12.1 & 1534.6 & 2.5 & 722.0 & 0.0 \\
\hline
\end{tabular}

En cuanto a la capacidad antioxidante ( $\mu \mathrm{mol}$ $\mathrm{TE} / 100 \mathrm{~g}$ muestra) de las tres accesiones de oca fresca varia de 722 a $1732(\mu \mathrm{mol}$ TE/100 g muestra), la oca purpura tiene una significativa $(P<$ $0.05)$ actividad antioxidante comparado a las otras accesiones, Chirinos et al. (2008) determinaron la capacidad antioxidante de mashua y obtuvieron 5.8 $\pm 0.9 \mu \mathrm{molTE} / \mathrm{g}$ mashua. Sin embargo después de la cocción se reduce en aproximadamente $99,3 \%$ de su actividad antioxidante, en forma similar ocurre en oca roja y en oca rosada se perdió por completo. Podemos señalar que la cocción por ebullición tiene un efecto drástico en esta propiedad funcional, probablemente se degradan los compuestos fenólicos, debido a que son termolábiles. La pérdida de capacidad antioxidante después del hervido ha sido observado en varios vegetales (Ismail et al. 2004; Zhang y Hamauzu, 2004), el cual estaría relacionado con la perdida de los compuestos fenólicos debido a una simple difusión en el agua de cocción. Sin embargo Natella et al. (2008) no observaron diferentes comportamientos de la capacidad antioxidante de alimentos después del hervido, la capacidad 
antioxidante del tomate (Halvorsen et al. 2002; Wu et al. 2004) y zanahoria (Halvorsen et al. 2006) después de la cocción se incrementó y es probable por la liberación de carotenoides en este tipo de vegetales. La preparación de alimentos puede incrementar la extractibilidad y disponibilidad de carotenoides por ruptura de la pared celular y el complejo caroteoide - proteína (Erdman et al. 1988; Mguyen y Schwartz 1998). Estas variaciones de propiedades funcionales de los alimentos puede incrementarse 0 reducirse dependiendo del tipo de cocción (microndas, a presión, horneado, etc.) y dependiendo del tipo de alimento y clase de compuesto bioactivo que predomina. Por lo tanto es importante tener en cuenta a la hora de elegir el método de cocción de los vegetales a fin de evitar pérdidas significativas de los compuestos fenólicos o carotenoides, ya que son compuestos valiosos que favorecen la salud del hombre. En la figura 6 se muestra los resultados de los cromatogramas de antocianidinas de oca púrpura, en el cual se observa 11 picos, siendo el pico con tiempo de retención de 8.44 min de mayor área y altura, lo que revela ser una antocianidina de mayor porcentaje, seguido por el pico de $4.54 \mathrm{~min}$. Estos picos representan los pigmentos predominantes en esta accesión. Asimismo, en el cuadro 3, se observa la descripción cuantitativa de cada pico en cuanto al tiempo de retención, área en mAU y porcentaje de área.

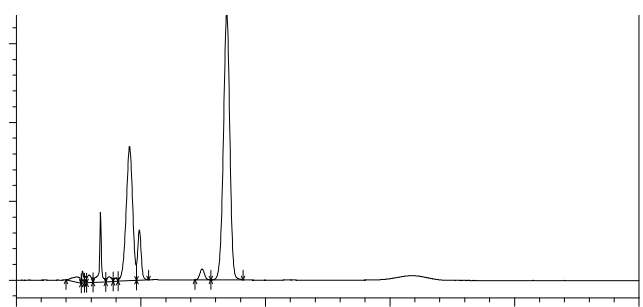

Figura 6. Cromatogramas de antocianidinas de ova púrpura fresca
Cuadro 3. Picos cromatográficos de oca púrpura fresca PDA Ch1 520nm 4nm

\begin{tabular}{lclccc}
\hline Peak\# & Ret. Time & Area & Heiqht & Area \% & Heiaht \% \\
\hline 1 & 2.421 & 14634 & 675 & 1.338 & 0.933 \\
2 & 2.654 & 7445 & 1524 & 0.681 & 2.105 \\
3 & 2.768 & 4189 & 775 & 0.383 & 1.071 \\
4 & 2.921 & 11068 & 966 & 1.012 & 1.335 \\
5 & 3.377 & 49428 & 8809 & 4.520 & 12.170 \\
6 & 3.729 & 7990 & 612 & 0.731 & 0.845 \\
7 & 3.979 & 3996 & 383 & 0.365 & 0.528 \\
8 & 4.544 & 307624 & 16986 & 28.133 & 23.469 \\
9 & 4.940 & 62088 & 6354 & 5.678 & 8.779 \\
10 & 7.453 & 18103 & 1395 & 1.656 & 1.928 \\
11 & 8.444 & 606902 & 33901 & 55.503 & 46.838 \\
Total & & 1093467 & 72380 & 100.000 & 100.000 \\
\hline & & & & & \\
\hline
\end{tabular}

\section{CONCLUSIÓN}

La oca púrpura fresca muestra un contenido significativo $(P<0.05)$ de polifenoles, antocianinas monoméricas y capacidad antioxidante en relación a las accesiones roja peruanita y rosada. La cocción por ebullición de las accesiones de oca disminuyó drásticamente al contenido de polifenoles y su actividad antioxidante. La preservación de los polifenoles y su propiedad funcional dependerá del tipo de alimento y método de cocción.

\section{REFERENCIAS BIBLIOGRÁFICAS}

1. Alvarez. A. Recursos genéticos del Centro de Investigación en Cultivos Andinos (CICA) de la Universidad del Cusco. III Congreso Internacional de Cultivos Andinos. La Paz, Bolivia, 1982.

2. Arbizu. C. y Robles, E. La colección de los cultivos de raíces y tubérculos andinos de la Universidad de Huamanga. Anales, $\mathrm{V}$ Congreso Internacional de Sistemas Agropecuarios Andinos, Puno, Perú. 1986.

3. Collazos, C. La composición de los alimentos peruanos. $5^{\circ}$ Edición. Ministerio de Salud. INS, Lima Perú, 1975.

4. Herquinio, F. y Toribio, L. Mantenimiento y evaluación de la colección internacional de oca. Anales, IV Congreso Internacional de cultivos andinos. Pasto, Colombia, 1984.

5. Jiménez, L. A. Población óptima de plantas de oca (Oxalis tuberosa Mol.) V Congreso Internacional de Sistemas Agropecuarios 
Andinos. PISA_CIID_ACDI. Puno, Perú, 1986

7. Astrid, G. G. Anthocyanins as natural colorants and Bioactive compounds. Acta biol. Colomb., Vol. 13 No. 3, 27 - 36, 2008

8. Zhenzhen Xua, B, C, Jihong Wua, B, C, Yan Zhanga, B, C, Xiaosong Hua, B, C, Xiaojun Liao, A, B, C, and Zhengfu Wanga, $B, C$. Extraction of anthocyanins from red cabbage using high pressure $\mathrm{CO} 2$ Bioresource Technology Volume 101, 18, 7151-7157, 2010.

9. Ines J. Seabraa, B, Mara Elga M. Bragaa, Maria Teresa Batista C, Herminio C. de Sousaa. Effect of solvent $\left(\mathrm{CO}_{2} /\right.$ ethanol/ $\left./ \mathrm{H}_{2} \mathrm{O}\right)$ on the fractionated enhanced solvent extraction of anthocyanins from elderberry pomace. J. of
Supercritical Fluids, 54, 145-152, 2010.

10. Cevallos-Casals BA, Cisneros-Zeballos L. Stability of Anthocyanin based Aqueous Extract of Andean Purple Corn and Red Fleshed Sweet Potato Compared to Synthetic and Natural Colorants. Food Chem. 2004;86:69-77.

11. Wrolstad R. E. Anthocyanin pigmentsbioactivity and coloring properties. J. Food Sci. 2004;69(5):C419-C425.

12. Zhishen, J.; Mengcheng, T.; Jianming, W. The determination of flavonoid contents in mulberry and their scavenging effects on superoxide radicals, Food Chemistry, 64 555559, 1999. 